\title{
Oral Mucosal Microvascular Abnormalities: An Early Marker of Bronchopulmonary Dysplasia
}

\author{
CLAUDIO DE FELICE, GIUSEPPE LATINI, STEFANO PARRINI, GIORGIO BIANCIARDI, \\ PAOLO TOTI, ROBERT J. KOPOTIC, AND DONALD M. NULL
}

\begin{abstract}
Neonatal Intensive Care Unit [C.D.F.], Azienda Ospedaliera Universitaria Senese, University of Siena, Viale Bracci 16, 53100 Siena, Italy; Division of Neonatology [G.L.], Perrino Hospital, Piazza Di Summa 72100, Brindisi, Italy; Clinical Physiology Institute [GL], National Research Council of Italy, Lecce Section, Italy; Department of Odontostomatologic Sciences [S.P.], University of Siena, Viale Bracci 16, 53100 Siena, Italy; Department of Human Pathology and Oncology [G.B., P.T.], University of Siena, Viale Bracci 16, 53100 Siena, Italy; Department of Pediatrics [R.J.K.], Obstetrics and Reproductive Medicine, University of Siena, Viale Bracci 16, 53100 Siena, Italy; ConMed Co. [R.J.K.], Vital Signs Development Center, San Juan Capistrano, CA 92675; and Division of Neonatology [D.M.N.], University of Utah Hospitals and Clinics, Salt Lake City, UT 84132
\end{abstract}

\begin{abstract}
An abnormal pulmonary vasculature has been reported as an important component of bronchopulmonary dysplasia (BPD). We tested the hypothesis of an early abnormal vascular network pattern in infants with BPD. Fifteen infants with BPD (nine boys and six girls; gestational age $27.5 \pm 2.0 \mathrm{wk}$; birth weight $850 \pm$ $125 \mathrm{~g}$ ) and 15 sex- and gestational age-matched infants (nine boys and six girls; gestational age $27.6 \pm 2.6 \mathrm{wk}$; birth weight $865 \pm 135 \mathrm{~g}$ ) were examined on postnatal days 1 and 28. BPD infants showed a significantly higher prevalence of histologic chorioamnionitis $(p=0.009)$, as well as higher intubation duration $(p=0.0004)$, oxygen supplementation $(p<0.0001)$, and initial illness severity $(p=0.0002)$ than the BPD-negative population. The lower gingival and vestibular oral mucosa was chosen as the study area. The blood vessel area was determined, and the oral vascular networks were characterized by analyzing their complexity ( $D$, at two scales: $D 1-46, D 1-15)$, tortuosity
\end{abstract}

\section{ABSTRACT}

(Dmin), and randomness (L-Z) of the vascular loops. Infants with BPD showed a significantly lower blood vessel area as well as a higher vascular network complexity ( $D$ 1-46, D 1-15, and L-Z) than control subjects $(p<0.0001)$. Our findings provide a new early clinical sign in BPD and stress the importance of an early disorder in the oral mucosal vascularization process in the disease pathogenesis. (Pediatr Res 56: 927-931, 2004)

\section{Abbreviations}

BPD, bronchopulmonary dysplasia

BVA, blood vessel area

D, fractal dimension

Dmin, minimum path fractal dimension

HCA, histologic chorioamnionitis

$\mathbf{L}-\mathbf{Z}$, relative Lempel-Ziv complexity
Bronchopulmonary dysplasia (BPD) remains an important cause of mortality and morbidity in preterm infants. BPD is infrequent in infants with birth weight $>1200 \mathrm{~g}$ or with gestation $>30 \mathrm{wk}$, whereas among infants with birth weight $<1000 \mathrm{~g}$, a prevalence of $\sim 30 \%$ has been reported $(1,2)$. To date, the pathogenesis of BPD remains unclear. Besides the well-known oxygen toxicity, volutrauma, and mechanical injury mechanisms (3), antenatal or postnatal inflammatory response $(4,5)$ and leakage of plasticizers from endotracheal tubes $(6,7)$ have been more recently associated with injury to the immature lung and/or the development of BPD. Delayed

Received December 5, 2003; accepted May 26, 2004

Correspondence: Claudio De Felice, M.D., Neonatal Intensive Care Unit, Azienda Ospedaliera Universitaria Senese, Policlinico "Le Scotte", Viale M. Bracci 16, I-53100 Siena, Italy; e-mail: defelice.claudio@libero.it

DOI: 10.1203/01.PDR.0000145259.85418.1D and disturbed formation of the terminal respiratory units have been reported in BPD (8), together with a disordered vascular development and a decreased production of angiogenic factors $(9,10)$. In the past two decades it has been realized that many biologic systems have no characteristic length scale, thus having fractal or self-affine properties (11). Fractals are of great value in biology, where surface phenomena are of crucial importance and a number of complex anatomic structures, including arterial and venous trees, also display fractal-like geometry. We tested the hypothesis of an abnormal mucosa vascularization and network pattern in BPD by using fractal analysis.

\section{METHODS}

Fifteen consecutive preterm infants with BPD (nine boys and six girls; gestational age $27.5 \pm 2.0 \mathrm{wk}$; birth weight 850 $\pm 125 \mathrm{~g}$ ) were examined, together with 15 sex- and gestational 
age-matched control infants without BPD (nine boys and six girls; gestational age $27.6 \pm 2.6 \mathrm{wk}$; birth weight $865 \pm$ $135 \mathrm{~g}$ ). A diagnosis of BPD was made on the basis of all of the following criteria (12): 1) requirement of intermittent positivepressure ventilation $\geq 3 \mathrm{~d}$ during the first week after birth, 2) clinical signs of chronic respiratory disease persistent for $>28$ $\mathrm{d}, 3$ ) requirement of supplemental oxygen for $>28 \mathrm{~d}$ to maintain an arterial partial pressure of oxygen $>50 \mathrm{~mm} \mathrm{Hg}$, and 4) typical changes on the chest radiograph. All of the participating infants were evaluated during the first day of life and at $28 \mathrm{~d}$. Initial severity of illness was assessed using the five-item scoring system Clinical Risk Index for Babies II (CRIB-II) (13). Accordingly, all infants were examined after informed parental consent, and the ethics committee approved the study.

Placental histology. Placental histologic examination was performed as previously described (14), and a diagnosis of histologic chorioamnionitis (HCA) was made given the presence of at least 10 polymorphonuclear leukocytes per field in 10 nonadjacent 400-power fields in membranes and/or placental chorionic plate. Pathologists were unaware of the neonatal outcomes (interobserver agreement in identifying histologic CA: $\kappa=0.97, \mathrm{SE}=0.015,95 \%$ CI $0.93-0.98$ ).

Oral mucosal vascular analysis. The lower gingival and vestibular oral mucosa was chosen as the study area, because of its accessibility. High-resolution photographs of the selected areas were acquired using a Yashica Dental Eye photocamera with an automated on-axis flashbulb and a 55-mm, f 1:4 Yashica lens. Kodak Elite Chrome 100 ISO/21 DIN films were used and developed according to the standard E-6 procedure (Fig. 1). Images were digitized using a Canon Canoscan FS2710 scanner (color resolution 680 dpi, $\times 6.67$ magnifica-
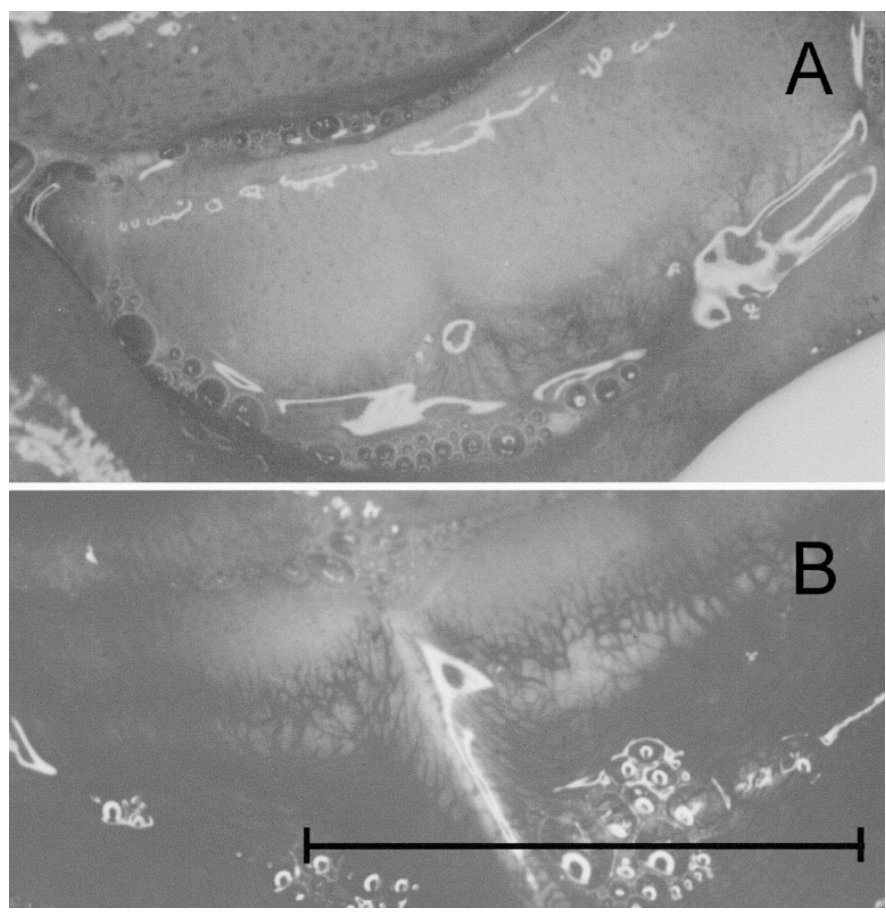

Figure 1. Original photographs of lower vestibular oral mucosa in a typical infant with BPD $(A)$ and in a gestational age-matched control neonate $(B)$. The size bar corresponds to $1 \mathrm{~cm}$. tion) with a Windows 98 operating system. The percentage blood vessel area (BVA\%) was determined by calculating the percentage area of the imaged oral mucosa covered by vascularization, using the Image Pro-Plus version 1.3 image analysis software (Image Pro-Plus-Media Cybernetics, Silver Spring, MD), with mean intra- and interobserver variability (coefficient of variation) of $2.5 \pm 1.8 \%$ and $5.6 \pm 3.2 \%$, respectively $(n=$ 6; Fig. 2). The obtained vascular network images were converted into binary skeletonized form for geometric pattern analysis. Manual outline of the two-dimensional (2-D) vascular network's trajectories was performed using Adobe Photoshop (Adobe Systems, San Jose, CA) on a Sony 19-inch Trinitron Multiscan G420 screen (16 $\mu \mathrm{m} /$ pixel resolution) by two operators who were unaware of the patients' study population. Nonreadable areas were $<5 \%$ of the total area. Images were processed to threshold the vessel network without background interference, and the networks were subsequently converted into an outline of single pixels (Fig. 3). The local fractal dimension $(D)$ was determined for two regions of box lengths $<740 \mu \mathrm{m}$ [pixels $1-46, D^{1-46}$ ] and $<140 \mu \mathrm{m}$ [pixels $1-15$, $\left.D^{1-15}\right]$, respectively. The fractal dimensions of 2-D skeletonized images were measured using the box-counting algorithm, according to the relation $\mathrm{N}(\mathrm{L}) \sim \mathrm{L}^{-D}$, where $\mathrm{L}$ is the box size, and $\mathrm{N}(\mathrm{L})$ is the number of squares (15). The measuring procedure was calibrated against shapes of known fractal dimension having an inaccuracy of $\pm 2 \%$.

Although the scaling plots (number of boxes versus box size, $\log$-log plots) for the examined microvascular networks at two different scales $\left[D^{1-15}, D^{1-46}\right]$ indicated a crossover between a uniform planar pattern $(\mathrm{D}=2$, approximate slope for small box sizes) and lines ( $\mathrm{D}=1$, approximate slope for large box sizes), thus suggesting that the networks are not properly fractal (data not shown), the box-counting method was maintained as a means to characterize the pattern geometry.

The fractal dimension of the minimum path, Dmin, was computed for each vascular cluster from the power law $l_{c}=$ $\mathrm{r}^{\mathrm{Dmin}}$, where Dmin is the exponent that governs the dependence of the minimum path length between two points $\left(l_{c}\right)$ on the Pythagorean distance ( $r$ ) between them in a fractal random material (16). After enlarging the image to $\times 2.4$ magnification, thinning to one pixel, and discarding all areas with a diameter less than pixels, the half perimeter $\left(\mathrm{x}_{\mathrm{i}}\right)$ and the maximum diameter $\left(\mathrm{y}_{\mathrm{i}}\right)$ of either the vessel loops or vessel-free areas in the single-pixel 2-D pattern were measured using an automated
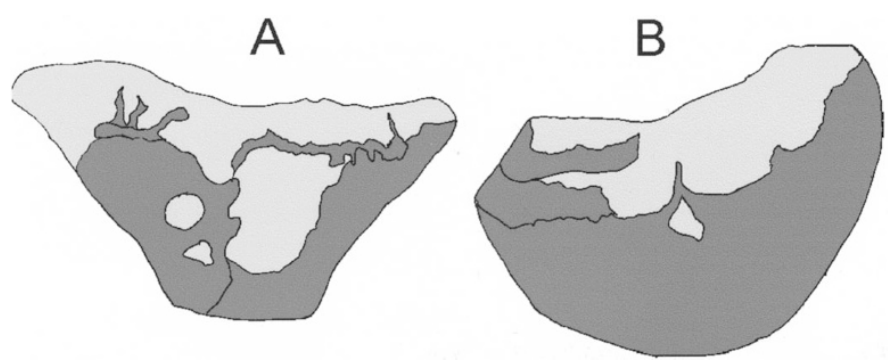

Figure 2. Schematic representation of the BVA\% as determined by calculating the percentage area of the imaged oral mucosa covered by vascularization. (A) BPD infant. (B) Control newborn. Red, vascularized areas; grey, avascular areas. 


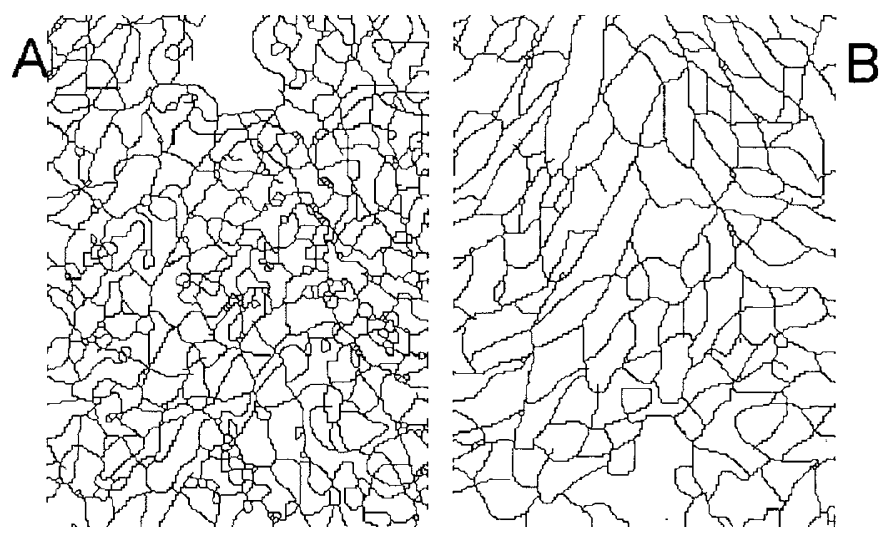

Figure 3. 2-D skeletonized microvascular networks in an infant with BPD $(A)$ compared with a gestational age-matched control subject $(B)$.

procedure (Image Pro-Plus version 1.3 image analysis software; examined areas for each sample: 500-1000). The slope of the Log/Log plot $\mathrm{x}_{\mathrm{i}} / \mathrm{y}_{\mathrm{i}}$ represented Dmin. The method was validated with the original one by Herrmann and Stanley (16) with a maximum shift of $\pm 3 \%$. To determine the algorithmic complexity of the vascular patterns, we calculated relative Lempel-Ziv (L-Z) (17) values according to the Kaspar and Schuster algorithm (18) using the Chaos Data Analyzer version 2.1 software package (CDA: Pro, The Academic Software Library, North Carolina State University, Raleigh, NC). It was accepted that relative $\mathrm{L}-\mathrm{Z}$ values could range from near 0 for a deterministic equation to $\sim 1.0$ for destructured random phenomena (i.e. sinus function L-Z 0.0044; white noise 1.047). The vascular network analysis was determined to be reproducible, given mean intra- and interobserver coefficients of variation of $\leq 5.0$ and $\leq 10 \%$, respectively.

Statistics. Variables were tested for normality using either the $\chi^{2}$ or Kolmogorov-Smirnov tests. Group sizes were calculated to give a $90 \%$ power to detect differences between group means of 1 SD of each normally distributed variable, with an $\alpha$ value of 0.05 . Differences among group means were analyzed by either the $t$ test, with $95 \%$ confidence intervals of differences of means between the groups, or the Wilcoxon's test, as appropriate. Associations between normally distributed, continuous variables were tested using univariate regression analysis. A two-sided $p<0.05$ was considered to indicate statistical significance, and the Bonferroni corrected significance $(p<0.05 / \mathrm{n})$ levels were used for multiple $t$ tests. The
MedCalc version 7.2.1.0 statistical software package (MedCalc Software, Mariakerke, Belgium) was used.

\section{RESULTS}

BPD infants showed a significantly higher prevalence of HCA $(p=0.009)$ than control infants (Table 1). BPD infants also showed a significantly higher intubation $(p=0.0004)$ and $\mathrm{O}_{2}$ supplementation duration $(p<0.0001)$, as well as higher Clinical Risk Index for Babies II scores $(p=0.0002)$ than the BPD-negative population. In contrast, the differences in proportion of endotracheal intubation ( $p=0.084)$, arterial partial pressure of carbon dioxide/fraction of inspired oxygen ratio $(p=0.34)$, and intubation duration/supplementary $\mathrm{O}_{2}$ duration $(p=0.33)$ were not statistically significant.

BPD infants on day 1 showed significantly lower BVA\% than control subjects $(35.05 \pm 8.86$ versus $65.31 \pm 12.43 \% ; p$ $<0.0001$ ), and their oral vascular networks showed significantly higher complexity $\left[D^{1-46} 1.92 \pm 0.036\right.$ versus $1.75 \pm$ $0.091(p<0.0001) ; D^{1-15} 1.29 \pm 0.072$ versus $1.17 \pm 0.032$ $(p<0.0001) ; \mathrm{L}-\mathrm{Z} 0.85 \pm 0.036$ versus $0.71 \pm 0.084(p<$ $0.0001)]$ than control subjects (Table 2). An inverse correlation between BVA\% and fractal dimension was observed (BVA\% versus $D^{1-46}: r=-0.60, p=0.01$; BVA\% versus $D^{1-15}: r=$ $-0.61, p=0.0088)$. Applying the receiver operating characteristic statistics, a $\mathrm{BVA} \% \leq 49.17 \%$ resulted in correctly classifying the subjects who belonged to the two groups (BPD patients versus control subjects) with $100 \%$ sensitivity and $100 \%$ specificity. Statistical differences observed on day 1 were maintained on day 28 (Table 3). Nine (60\%) of the BPD patients were still on oxygen supplementation at $36 \mathrm{wk}$ postconceptional age.

A pilot study on oral vascular variables in HCA infants without BPD ( $n=20 ; 11$ boys and nine girls; gestational age $27.6 \pm 2.2$; birth weight $845 \pm 130 \mathrm{~g}$ ) showed results comparable to HCA-negative infants ( $n=20 ; 10$ boys and 10 girls; gestational age $27.8 \pm 2.1$; birth weight $850 \pm 140 \mathrm{~g}$ ) without BPD [BVA\%: $60.7 \pm 8.6$ versus $67.61 \pm 14.08(p=0.12)$; $D^{1-46}: 1.76 \pm 0.072$ versus $1.74 \pm 0.10(p=0.67) ; D^{1-15}$ : $1.17 \pm 0.035$ versus $1.18 \pm 0.031(p=0.73) ; D$ min: $0.99 \pm$ 0.055 versus $1.00 \pm 0.026(p=0.80) ; \mathrm{L}-\mathrm{Z}: 0.74 \pm 0.063$ versus $0.70 \pm 0.094(p=0.13)]$.

Table 1. Histologic chorioamnionitis, assisted ventilation, and initial illness severity in extremely low birth weight infants with and without BPD-28 days

\begin{tabular}{lcc}
\hline \multicolumn{1}{c}{ Variables } & BPD $+(n=15)$ & BPD- $(n=15)$ \\
\hline Antenatal steroids & $14(93.3 \%)$ & $14(93.3 \%)$ \\
Endotracheal intubation & $14(93.3 \%)$ & $9(60 \%)$ \\
Intubation duration (h) & $456(356-745)$ & $24(9-41) \dagger$ \\
Supplementary $\mathrm{O}_{2}$ duration $(\mathrm{h})$ & $1210(903-1402)$ & $48(24-72) \dagger$ \\
Intubation time $/ \mathrm{O}_{2}$ time ratio & $0.41(0.29-0.58)$ & $0.51(0.24-0.62)$ \\
$\mathrm{PaO}_{2} / \mathrm{FIO}_{2}$ ratio & $1.30 \pm 0.78$ & $1.74 \pm 1.57$ \\
$\mathrm{Histologic}$ chorioamnionitis & $13(86.7 \%)$ & $5(33.3 \%)^{*}$ \\
CRIB II & $12.71 \pm 2.85$ & $7.8 \pm 3.42 \dagger$ \\
\hline
\end{tabular}

$\mathrm{PaO}_{2}$, arterial partial pressure of oxygen; $\mathrm{FIO}_{2}$, fraction of inspired oxygen; CRIB II, Clinical Risk Index for Babies II.

$* p<0.01$.

$\dagger p<0.0001$. 
Table 2. Comparisons of oral vascular mucosal variables at day 1

\begin{tabular}{lcccc}
\hline Variable & $\begin{array}{c}\text { BPD } \\
(n=15)\end{array}$ & $\begin{array}{c}\text { Controls } \\
(n=15)\end{array}$ & $\begin{array}{c}95 \% \text { CI of } \\
\text { Difference }\end{array}$ & $p$ value \\
\hline BVA \% & $35.05(8.86)$ & $65.31(12.43)$ & 22.19 to 38.33 & $<0.0001$ \\
$\mathrm{D}^{1-46}$ & $1.92(0.036)$ & $1.75(0.091)$ & 0.12 to 0.22 & $<0.0001$ \\
$\mathrm{D}^{1-15}$ & $1.29(0.072)$ & $1.17(0.032)$ & 0.078 to 0.16 & $<0.0001$ \\
Dmin & $1.02(0.038)$ & $1.00(0.035)$ & -0.012 to 0.042 & 0.26 \\
$\mathrm{~L}-\mathrm{Z}$ & $0.85(0.036)$ & $0.71(0.084)$ & 0.089 to 0.18 & $<0.0001$ \\
\hline
\end{tabular}

Values are expressed as means (SD) and confidence interval (CI) of the differences between the two study groups.

Table 3. Comparisons of oral vascular mucosal variables at day 28

\begin{tabular}{lcccc}
\hline Variable & $\begin{array}{c}\text { BPD } \\
(n=15)\end{array}$ & $\begin{array}{c}\text { Controls } \\
(n=15)\end{array}$ & $\begin{array}{c}\text { 95\% CI of } \\
\text { Difference }\end{array}$ & $p$ value \\
\hline BVA \% & $41.45(5.78)$ & $66.28(16.47)$ & 15.49 to 33.95 & $<0.0001$ \\
$\mathrm{D}^{1-46}$ & $1.90(0.017)$ & $1.76(0.058)$ & 0.11 to 0.18 & $<0.0001$ \\
$\mathrm{D}^{1-15}$ & $1.26(0.075)$ & $1.17(0.037)$ & 0.049 to 0.14 & $<0.002$ \\
Dmin & $1.03(0.048)$ & $1.01(0.037)$ & -0.0051 to 0.059 & 0.096 \\
L-Z & $0.85(0.019)$ & $0.69(0.10)$ & 0.11 to 0.22 & $<0.0001$ \\
\hline
\end{tabular}

Values are expressed as means (SD) and CI of the differences between the two study groups.

\section{DISCUSSION}

The main findings of the present study indicate the presence of a previously unrecognized early disorder of reduced oral mucosal vascularization process in infants who subsequently develop BPD. The lower gingival and vestibular oral mucosa proved a readily accessible site for analysis of BVA\%. The findings of a significantly lower BVA\% by the first postnatal day support the hypothesis of a key role for early vascular abnormalities in the pathogenesis of BPD and seem to confirm that BPD may begin in intrauterine life $(4,5)$. We have found that these abnormalities are not limited to the lung, but the BPD-associated oral microvessel networks exhibit a significantly increased overall complexity at both larger and smaller scales, together with an increased destructured randomness. The $D^{1-46}$ results for the vascular networks of the BPD infants display intermediate characteristics between the diffusionlimited aggregation behavior of control networks and the percolation-like scaling of tumor-associated vascular networks (19). In contrast, vessel tortuosity was comparable between the groups. Although the mechanisms underlying this clinical observation remain to be clarified, angiostatic agents $(9,20)$ may play a previously unrecognized, major role in producing injury to the developing lung. Moreover, an abnormal pulmonary vasculature has been reported as an important component of BPD $(9,10)$. The absence of microvascular changes in HCA patients who subsequently will not develop BPD confirms the multifactorial pathogenesis of the disease where other factors may also play a role, including mechanical trauma and oxygen toxicity (3-5), pulmonary edema associated with patent ductus arteriosus and/or excess fluid administration (21), nutritional deficiencies (22), a predisposition to airway reactivity (23), early adrenal insufficiency (24), and di-(2-ethylhexyl)phthalate release from endotracheal tubes $(6,7)$. Recent preliminary research indicates a reduced expression of vascular endothelial growth factor receptor 1 (25) in fetal capillaries in preterm placentas with chorioamnionitis, which is in line with the findings of altered vascularization in infants who have HCA and subsequently develop BPD. The correlation between antenatal infection/inflammation (i.e. HCA) and $\mathrm{BPD} /$ chronic lung disease has been well documented $(26,27)$. However, a significant interaction between chorioamnionitis and mechanical ventilation and/or postnatal sepsis in the pathogenesis of chronic lung disease has been reported (28), and the importance of ventilation strategy on the pulmonary outcome of premature infants has been stressed (29).

Molecular biology offers ever-increasing spectacular successes, although a detailed inventory of genes, proteins, and metabolites is not itself sufficient to understand biologic complexity (30). Elucidating the fractal and nonlinear mechanisms involved in physiologic control and complex signaling networks is emerging as a major challenge in the postgenomic era (31). In particular, for medical images, fractals geometry is increasingly used as an index of irregularity, thus evaluating angiogenic processes (32). To deliver oxygen and nutrients to all of the cells of the body, blood vessels must have fractal properties (33), and their branching pattern is very similar to the growth model called diffusion-limited aggregation. Furthermore, it has previously been shown that fractal dimension $(D)$ and other fractal characteristics can be used to identify abnormal vascular patterns, including corneal (34), retinal (35), and tumor-associated neovascular growth $(19,36)$. The precise mechanisms underlying the observed increased vascular complexity remain unclear, although extracellular matrix is known to play an important role in angiogenesis and blood vessel geometry $(37,38)$, and changes in extracellular matrix components have been previously reported in experimental models and patients with BPD $(39,40)$. Although study of larger populations should be performed to confirm our preliminary observations, the findings of early changes in the oral mucosal vascularization associated with BPD may be crucial in selecting candidate patients for treatment as specific treatments become available regarding vasculogenesis.

\section{REFERENCES}

1. Bancalari E, Gonzales A 2000 Clinical course and lung formation abnormalities during development of neonatal bronchopulmonary dysplasia. In Bland RD, Coalson JJ (eds) Bronchopulmonary Dysplasia in Early Infancy. Marcel Dekker, New York, pp 41-64

2. Jobe AH, Bancalari E 2001 Bronchopulmonary dysplasia. Am J Respir Crit Care Med 163:1723-1729

3. Rojas MA, Gonzalez A, Bancalari E, Claure N, Poole C, Silva-Neto G 1995 Changing trends in the epidemiology and pathogenesis of neonatal chronic lung disease. J Pediatr 126:605-610

4. Yoon BH, Romero R, Kim KS, Park JS, Ki SH, Kim BI, Jun JK 1999 A systemic fetal inflammatory response and the development of bronchopulmonary dysplasia. Am J Obstet Gynecol 181:773-779

5. Schmidt B, Cao L, Mackensen-Haen S, Kendziorra H, Klingel K, Speer CP 2001 Chorioamnionitis and inflammation of the fetal lung. Am J Obstet Gynecol 185:173177

6. Latini G, Avery GB 1999 Materials degradation in endotracheal tubes: a potential contributor to bronchopulmonary dysplasia. Acta Paediatr 88:1174-1175

7. Magliozzi R, Nardacci R, Scarsella G, Di Carlo V, Stefanini S 2003 Effects of the plasticiser DEHP on lung of newborn rats: catalase immunocytochemistry and morphometric analysis. Histochem Cell Biol 120:41-49

8. Margraf LR, Tomashefski JF, Bruce MT, Dahms BB 1991 Morphometric analysis of the lung in bronchopulmonary dysplasia. Am Rev Respir Dis 143:391-400

9. D'Angio CT, Maniscalco WM 2002 The role of vascular growth factors in hyperoxiainduced injury to the developing lung. Front Biosci 7:d1609-d1623

10. Bhatt AJ, Pryhuber GS, Huyck H, Watkins RH, Metlay LA, Maniscalco WM 2001 Disrupted pulmonary vasculature and decreased vascular endothelial growth factor, Flt-1, and TIE-2 in human infants dying with bronchopulmonary dysplasia. Am J Respir Crit Care Med 164:1971-1980 
11. Goldberger AL, Rigney DR, West BJ 1990 Chaos and fractals in human physiology. Sci Am 262:42-49

12. De Felice C, Latini G, Del Vecchio A, Toti P, Bagnoli F, Petraglia F 2002 Smal thymus at birth: a predictive radiographic sign of bronchopulmonary dysplasia. Pediatrics 110:386-388

13. Parry G, Tucker J, Tarnow-Mordi W; UK Neonatal Staffing Study Collaborative Group 2003 CRIB II: an update of the clinical risk index for babies score. Lancet 361:1789-1791

14. Toti P, De Felice C, Stumpo M, Schurfeld K, Di Leo L, Vatti R, Bianciardi G, Buonocore G, Seemayer TA, Luzi P 2000 Acute thymic involution in fetuses and neonates with chorioamnionitis. Hum Pathol 31:1121-1128

15. Peitgen HO, Jurgens H, Saupe D 1992 Chaos and Fractals. New Frontiers of Science. Springer-Verlag, New York

16. Herrmann HJ, Stanley HE 1988 The fractal dimension of the minimum path in twoand three-dimensional percolation. J Phys A Math Gen 21:L829-L833

17. Ziv J, Lempel A 1997 A universal algorithm for sequential data compression. IEEE Trans Inform Th 23:337-343

18. Kaspar F, Schuster HG 1987 Easily calculable measure for the complexity of spatio-temporal patterns. Phys Rev A 36:842-848

19. De Felice C, Latini G, Bianciardi G, Parrini S, Fadda GM, Marini M, Laurini RN, Kopotic RJ 2003 Abnormal vascular network complexity: a new phenotypical marke in heredity non-polyposis colorectal cancer family syndrome. Gut 12:1764-1767

20. Kallapur SG, Jobe AH, Ikegami M, Bachurski CJ 2003 Increased IP-10 and MIG expression after intra-amniotic endotoxin in preterm lamb lung. Am J Respir Crit Care Med 167:779-786

21. Gerhardt T, Bancalari E 1980 Lung compliance in newborns with patent ductus arteriosus before and after surgical ligation. Biol Neonate 38:96-105

22. Sosenko IR, Kinter MT, Roberts RJ 2000 Nutritional issues in chronic lung disease of premature infants. In: Bland RJ, Coalson JJ (eds) Chronic Lung Disease in Early Infancy. Marcel Dekker, New York, pp 285-296

23. Goldman SL, Gerhardt T, Sonni R, Feller R, Tapia JL, Bancalari E 1993 Early prediction of chronic lung disease by pulmonary function testing. J Pediatr 102:613617

24. Watterberg KL, Scott SM 1995 Evidence of early adrenal insufficiency in babies who develop bronchopulmonary dysplasia. Pediatrics 95:120-125

25. Kramer BW, Kämmerer U, Berg D, Groneck P, Speer CP 2003 Expression of heat shock protein 70 is increased but vascular endothelial growth factor receptor 1 is decreased in fetal capillaries in placentas from preterm deliveries with chorioamniositis. Pediatr Res 54:579
26. Schmidt B, Cao L, Mackensen-Haen S, Kendziorra H, Klingel K, Speer CP 2001 Chorioamnionitis and inflammation of the fetal lung. Am J Obstet Gynecol 185:173177

27. Speer CP 2003 Inflammation and bronchopulmonary dysplasia. Semin Neonatol 8:29-38

28. Van Marter LJ, Dammann O, Allred EN, Leviton A, Pagano M, Moore M, Martin C Developmental Epidemiology Network Investigators 2002 Chorioamnionitis, mechanical ventilation, and postnatal sepsis as modulators of chronic lung disease in preterm infants. J Pediatr 140:171-176

29. Latini G, De Felice C, Presta G, Rosati E, Vacca P 2003 Minimal handling and bronchopulmonary dysplasia in extremely low-birth-weight infants. Eur J Pediat 162:227-229

30. Oltvai ZN, Barabasi AL 2002 Systems biology. Life's complexity pyramid. Science 298:763-764

31. Goldberger AL, Amaral LA, Hausdorff JM, Ivanov PCh, Peng CK, Stanley HE 2002 Fractal dynamics in physiology: alterations with disease and aging. Proc Natl Acad Sci USA 99(suppl 1):2466-2472

32. Oczeretko E, Juczewska M, Kasacka I 2001 Fractal geometric analysis of lung cance angiogenic patterns. Folia Histochem Cytobiol 39(suppl 2):75-76

33. Tsonis AA, Tsonis PA 1987 Fractals: a new look at biological shape and patterning. Perspect Biol Med 30:355-361

34. Daxer A, Ettl A 1995 Corneal vascularization and its relation to the physical properties of the tissue: a fractal analysis. Curr Eye Res 4:263-268

35. Daxer A 1993 The fractal geometry of proliferative diabetic retinopathy: implications for the diagnosis and the process of retinal vasculogenesis. Curr Eye Res 2:11031109

36. Gazit Y, Berk DA, Leunig M, Baxter LT, Jain RK 1995 Scale-invariant behavior and vascular network formation in normal and tumor tissue. Phys Rev Lett 75:2428-2431

37. Suwa N 1981 Supracellular structural principle and geometry of blood vessels. Virchows Arch A Pathol Anat Histol 390:161-179

38. Distler O, Neidhart M, Gay RE, Gay S 2002 The molecular control of angiogenesis. Int Rev Immunol 21:33-49

39. Cederqvist K, Sorsa T, Tervahartiala T, Maisi P, Reunanen K, Lassus P, Andersson S 2001 Matrix metalloproteinases-2, -8, and -9 and TIMP-2 in tracheal aspirates from preterm infants with respiratory distress. Pediatrics 108:686-692

40. Kaarteenaho-Wiik R, Kinnula VL, Herva R, Soini Y, Pollanen R, Paakko P 2002 Tenascin-C is highly expressed in respiratory distress syndrome and bronchopulmonary dysplasia. J Histochem Cytochem 50:423-431 